\title{
Porous Silicon as Soft Material in Low-Frequency MEMS (MicroElectro-Mechanical Systems) Resonators
}

\author{
C. Malhaire1, A. Danescu², V. Lysenko1, A. Sabac ${ }^{1}$ \\ ${ }^{1}$ Université de Lyon, Institut de Nanotechnologies de Lyon INL-UMR 5270, CNRS, INSA Lyon, Villeurbanne, France \\ ${ }^{2}$ Université de Lyon, Institut de Nanotechnologies de Lyon INL-UMR 5270, CNRS, École Centrale de Lyon, Écully, France \\ Email: christophe.malhaire@insa-lyon.fr
}

How to cite this paper: Malhaire, C., Danescu, A., Lysenko, V. and Sabac, A. (2018) Porous Silicon as Soft Material in LowFrequency MEMS (MicroElectro-Mechanical Systems) Resonators. World Journal of Mechanics, 8, 431-443.

https://doi.org/10.4236/wjm.2018.811031

Received: September 7, 2018

Accepted: November 4, 2018

Published: November 7, 2018

Copyright ( $) 2018$ by authors and Scientific Research Publishing Inc. This work is licensed under the Creative Commons Attribution International License (CC BY 4.0).

http://creativecommons.org/licenses/by/4.0/

\begin{abstract}
This study focuses on the mechanical response of silicon on porous silicon bilayer cantilevers ended with a seismic mass. The porous silicon is intended to provide an alternative to decrease the cantilever stiffness for low-frequency MEMS applications. The first eigenfrequency of the cantilever is obtained using static deflection obtained under classical Euler-Bernoulli assumptions and Rayleigh method. In order to estimate the errors due to small-strain approximation and Euler-Bernoulli theory, the analytical results were validated through 3D finite element simulations for different cantilever geometries and porosities. Both bulk silicon and silicon on porous silicon bilayer cantilevers ended with a seismic mass were fabricated and we measured the first eigenfrequency $\left(f_{0}\right)$ and quality factor $(Q)$ by using a laser Doppler vibrometer. In agreement with the theoretical predictions we found that, when compared to bulk silicon cantilevers, the first eigenfrequency of a bilayer cantilever containing $6 \%$ porous silicon (at $50 \%$ porosity) on $94 \%$ bulk silicon is lowered by $5 \%$, from $(5447 \pm 120) \mathrm{Hz}$ to $\approx 5198 \mathrm{~Hz}$. This decrease is also accompanied by a reduction of the quality factor by two.
\end{abstract}

\section{Keywords}

Porous Silicon, Cantilever, Resonator

\section{Introduction}

The concept of energy harvesting gained new relevance since the recent development of ultra low-power embedded electronic devices. Energy harvesting from ambient vibrations enables new exciting opportunities for low power nano- 
and microsystems (MEMS) and line-powered motor-driven machines are excellent vibration sources to harvest from, with a frequency component of line power frequency at 60 (or 50 in non-U.S. countries) and twice-line-frequency at 120 (and 100 in non-U.S. countries). A variety of vibration sources are present in the environment and their typical frequency range of interest is $60-200$ [1]. Thus, a first challenge is the design of miniaturized resonators able to reach such low frequencies, and this is particularly difficult since eigenfrequencies tend to increase with shrinking dimensions and mass. Moreover, in order to use electro-active materials in thin films, a second challenge in device miniaturization is to preserve the good intrinsic coupling between these materials. The maximum power one can expect to retrieve depends on this coupling and on the quality factor of the harvesting device.

The classical inertial generator consists of a thin cantilever ended by a seismic mass (also called proof mass) and is designed to convert, via a transduction mechanism, a part of the kinetic energy present in the ambient vibrations into electrical energy. Transmitted through the frame of the device to the suspended mass, ambient vibrations represent external excitations and the elastic strains in the cantilever can be converted to generate electricity by means of a classical piezoelectric material [2].

This study focuses on the silicon cantilever and, in particular, on the use of porous silicon (PS) as an alternative to decrease the cantilever stiffness [3]. Since making a resonator with a low eigenfrequency can be a challenge even using a large seismic mass, we shall examine here only the simplest situation of a cantilever without both the electrode and the electro-active material. A versatile material, porous silicon, can display different morphologies by varying the formation parameters and doping level of the substrate [4]. Its high reactivity and high specific surface make it a good candidate for sensors based on electrical or electromechanical devices. Previous tentatives of integration of PS on silicon cantilevers were already reported in the literature for the development of chemical sensors. The formation of a thin, 100 thick surface layer of PS on silicon micro-cantilevers by vapor phase strain etching was presented by S. Stolyarova et al. [5]. For present purposes anodization of silicon [6] seems more appropriate since it works well up to very thick layers (up to substrate thickness). With such process, Garel et al. [7] reported Si/n-type porous-Si (6/5) bilayer cantilevers with eigenfrequency $f_{0}=385.71 \mathrm{kHz}$ and quality factor $Q=760$.

The paper is organized as follows: the second section gives a brief overview of the mechanical properties of PS with a particular accent on the interplay between the stiffness of PS and the first eigenfrequency of the cantilever. The third section presents the design of the cantilever and, for completeness, the analytical formulas for eigenfrequencies in the classical framework of multilayered beams under Euler-Bernoulli assumptions. We also provide a comparison between the analytical formulae and finite elements computation results in the fully threedimensional framework in finite strains. These results comfort the analytical 
predictions and confirm that the intrinsic geometric nonlinearities of the fully three-dimensional problem including the seismic mass can be neglected. The fourth section explains the samples preparation and the method used to measure eigenfrequencies. The mechanical response measured on a series of samples shows that, as expected, the first eigenfrequency follow closely the predicted effect and the use of PS allows to decrease the cantilever stiffness.

\section{Preliminaries}

\subsection{Elasticity of Porous Silicon}

Young's moduli for different values of porosity for porous silicon layers were investigated by X-ray diffraction, acoustic techniques, nanoindentation and Brillouin scattering. The obtained values are summarized and discussed in [8]. A good approximation for the Young's modulus in a $\mathrm{p}^{+}$-type PS material containing three-dimensional arrangement of open pores is given by

$$
E_{p}=C E_{b} \rho_{r}^{2}=C E_{b}(1-p)^{2}
$$

where $E_{p}$ and $E_{b}$ stand for the Young's moduli of porous and bulk silicon, respectively, $C$ is a constant including all the geometric scaling factors, $\rho_{r}$ is the relative density and $p$ the porosity. This approximation is consistent with the literature [9] [10] and was confirmed in [11] in the particular case of nano-porous silicon containing pores with cubic symmetry. Obtained from a discrete model including near-neighbors $(\mathrm{NN})$ and next-to-near-neighbors (NNN) interactions the model proposed in [11] fits particularly well previous mentioned experimental results. Moreover, the model is able to predict the more complex anisotropic behavior in situations where, due to directional anodization, the resulting macroscopic PS is strongly anisotropic. Back to the cubic case, it has been shown in [11] that the shear modulus and the Poisson ratio obey

$$
G_{p}=G_{b}(1-p)^{4}, \quad v_{p}=v_{b}(1-p) .
$$

\subsection{Porous Silicon for a Low Frequency Resonator}

Let us consider a simple cantilever clamped at one end without any seismic mass. Denoting by $L$ the cantilever length, by $w$ and $t$ the width and the thickness of its cross section respectively, by $\rho_{b}$ the mass density and $E_{b}$ the Young's modulus of the bulk silicon, the first eigenfrequency is given by

$$
f_{b}=\frac{\alpha^{2} t}{4 \pi L^{2}} \sqrt{\frac{E_{b}}{3 \rho_{b}}},
$$

where $\alpha \simeq 1.875$ is the first positive solution of the (characteristic) equation $\cos \alpha=-1 / \cosh \alpha$. For a PS cantilever with the same geometry, as the Young's modulus follows the rule given in (1) but $\rho_{p}=\rho_{b}(1-p)$ we obtain

$$
f_{p}=f_{b} \sqrt{1-p} \text {. }
$$


It follows that using porous silicon, one can decrease the eigenfrequencies of the cantilever and this remark was the starting point of our study. For technological reasons (fabrication limitations) the cantilevers we studied were merely two-layer materials: Si/PS at various thicknesses. For that case the derivation of eigenfrequencies and comparison with numerical values provided by finite element method computations under more general assumptions (finite strains in the fully three-dimensional framework) will be presented in the next section.

\subsection{Geometry of the Bilayer Cantilever}

In a fully functional energy-harvesting device, a bottom electrode, a piezoelectric material and a top electrode will cover the upper side of the cantilever. In order to investigate the mechanical behavior of the harvesting device we shall focus in the following only on a Si/PS cantilever and a generic geometry of the structure is illustrated in Figure 1.

\section{Analytical Results and Comparison with Finite Element Results}

\subsection{Neutral Axis and Flexural Stiffness of a Bilayer Cantilever}

In a bilayer beam, the position of the neutral axis, denoted $z_{0}$ and measured from the bottom face of the cantilever, is given by

$$
z_{0}=\frac{E_{b} t_{b}\left(t_{p}+t_{b} / 2\right)+E_{p} t_{p}^{2} / 2}{E_{b} t_{b}+E_{p} t_{p}}=\frac{E_{p} t_{p}^{2}+E_{b} t_{b}\left(t_{b}+2 t_{p}\right)}{2\left(E_{b} t_{b}+E_{p} t_{p}\right)},
$$

where $t_{p}$ and $t_{b}$ are the thicknesses of porous (bottom) layer and bulk (upper) layer, respectively. Using $I_{b}=w t_{b}^{3} / 12$ and $I_{p}=w t_{p}^{3} / 12$ for the quadratic moment of inertia with respect to their centroid axis for the bulk and porous layers respectively, the flexural stiffness of the bilayer, further denoted $E I$ can be computed as

$$
\begin{aligned}
E I & =E_{b}\left(I_{b}+w t_{b}\left(t_{p}+t_{b} / 2-z_{0}\right)^{2}\right)+E_{p}\left(I_{p}+w t_{p}\left(z_{0}-t_{p} / 2\right)^{2}\right) \\
& =\frac{w}{12} \frac{\left(E_{b} t_{b}^{2}+E_{p} t_{p}^{2}\right)^{2}+4 E_{b} E_{p} t_{b} t_{p}\left(t_{b}^{2}+t_{b} t_{p}+t_{p}^{2}\right)}{E_{b} t_{b}+E_{p} t_{p}} .
\end{aligned}
$$

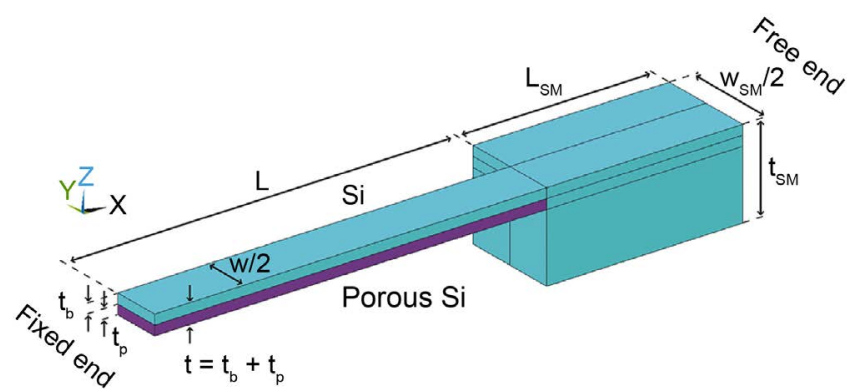

Figure 1. Design of the two-layer Si/Porous Si cantilever with seismic mass (half structure). 
As expected, the last relation, symmetric with respect to interchange of subscripts $p$ and $b$, reduces to $E I=E w\left(t_{b}+t_{p}\right)^{3} / 12$ in the case of equal elastic moduli.

\subsection{Static Deformation}

For completeness, we shall present in this subsection analytical results for the deflection of a generic bilayer cantilever in two cases (a) a first one when when the cantilever is loaded by a point load at its end and the mass of the cantilever is neglected (b) a second case that generalizes the first one by including the effect of its own weight and that of the seismic mass.

Denoting by $F$ the load due to the seismic mass and assuming that: 1) this load acts at $x=L+L_{S M} / 2$ and 2) the cantilever is rigid for $x>L$, the classical Euler-Bernoulli theory gives the static vertical deflection of the massless cantilever as

$$
d=\frac{F}{12 E I}\left\{\begin{array}{lc}
x^{2}\left[3 L_{S M}+2(3 L-x)\right] & 0 \leq x \leq L \\
L\left[6\left(L+L_{S M}\right) x-L\left(2 L+3 L_{S M}\right)\right] & L \leq x \leq L+L_{S M}
\end{array}\right.
$$

If the mass of the bilayer cantilever cannot be neglected (this is the case of a thick beam) assuming an uniform load $q=-g\left(\rho_{b} t_{b}+\rho_{p} t_{p}\right) w$ the vertical deflection obtained is given by

$d=\frac{1}{24 E I}\left\{\begin{array}{lc}{\left[q x^{2}-4(q L+F) x+6\left(q L^{2}+F\left(2 L+L_{S M}\right)\right)\right] x^{2}} & 0 \leq x \leq L \\ L\left[\left(4 q L^{2}+12 F\left(L+L_{S M}\right)\right) x-L\left(q L^{2}+2 F\left(2 L+3 L_{S M}\right)\right)\right] & L \leq x \leq L+L_{S M}\end{array}\right.$

In both cases, for $L<x<L+L_{S M}$, the seismic mass is assumed to be rigid so that the deflection for $x>L$ is completely determined by the values of $d(L)$ and $d^{\prime}(L)$ obtained for $x \leq L$.

In order to estimate the effect of the cantilever bending on a thin piezo-layer one can compute the axial strain at the upper surface. The longitudinal deformation along the beam, at a distance $h$ from the neutral axis, is obtained as $\varepsilon_{x x}=-\kappa h$, where the (linearized) curvature of the cantilever is given by $\kappa=\partial^{2} d / \partial x^{2}$. At the upper free-surface of the cantilever, we obtain

$$
\epsilon_{x x}=-\left(t_{b}+t_{p}-z_{0}\right) \frac{\partial^{2} d}{\partial x^{2}} .
$$

\subsection{Estimate for the First Eigenfrequency}

The fundamental frequency of the massless cantilever beam with a concentrated mass at $x=L+L_{S M} / 2$ (see notations in Figure 1) is given by [12]

$$
f_{0}=\frac{1}{2 \pi}\left(\frac{3 E I}{M_{S M} L^{3}(1+\eta / 2)^{3}}\right)^{1 / 2},
$$

where $\eta$ represents the non-dimensional number: $\eta=L_{S M} / L$. The result overestimates the first eigenfrequency of the cantilever since in contrast to our 
previous assumptions, in (10) one does not assume that the part of the beam located between $L$ and $L+L_{S M} / 2$ is rigid.

An alternative approach consists in using the solution for the cantilever static deflection in order to provide a better estimate (more exactly, an overestimate of it [13]) of the first eigenfrequency by using the Rayleigh principle. A similar approach was successfully used to calculate the eigenfrequency of a silicon accelerometer [14]. Using (7), neglecting the rigidity of the cantilever for $x>L$ and assuming that the seismic mass is much larger than the cantilever mass, the first eigenfrequency of the system including the seismic mass located at $x=L+L_{S M} / 2$ is given by

$$
f_{0}=\frac{1}{2 \pi}\left(\frac{E I}{M_{S M} L^{3}} \frac{12\left(4+6 \eta+3 \eta^{2}\right)}{\left(16+48 \eta+63 \eta^{2}+42 \eta^{3}+12 \eta^{4}\right)}\right)^{1 / 2} .
$$

As an example, if $t_{b}=18 \mu \mathrm{m}, t_{p}=2 \mu \mathrm{m} \quad$ (i.e., only $10 \%$ of the total cantilever thickness is a PS layer) at $50 \%$ porosity, if the bilayer dimensions are $L=2 \mathrm{~mm}, w=500 \mu \mathrm{m}$ and seismic mass dimensions are $L_{S M}=w_{S M}=1 \mathrm{~mm}$ and $t_{S M}=450 \mu \mathrm{m}$, Equation (11) leads to $f_{0}=454 \mathrm{~Hz}$ for the bilayer cantilever. Same dimensions for a bulk silicon cantilever $\left(t_{b}=20 \mu \mathrm{m}, t_{p}=0 \mu \mathrm{m}\right)$ provide $f_{0}=507 \mathrm{~Hz}$, so that porosification of $10 \%$ of the cantilever thickness decreases the first eigenfrequency of about $10 \%$. We conclude that even a relatively thin PS layer could lead to a measurable change in the first eigenfrequency of the structure.

Before fabrication and discussion of the experimental results, one must ensure of the reliability of the given analytical models in the case of some common geometries found in MEMS devices, under actual simplifying assumptions (one dimensional model for bending under Euler-Bernoulli hypothesis, neglecting the rigidity of the cantilever for $x>L$ and accounting only for the seismic mass) can be confirmed in a more general framework (finite strains, three-dimensional elasticity). For this reasons the next subsection compares the analytical results for the deflection, surface strain and first eigenfrequency for different bilayer cantilevers to finite element simulations of fully three-dimensional structures in finite strains.

\subsection{FEM Results and Comparison with Analytical Estimates}

Finite element simulations were performed using Ansys 14 and the three-dimensional model of the cantilever was built using 3D, 20-nodes, structural solid (SOLID186) elements. These elements have three degrees of freedom per node (translations in the nodal $\mathrm{x}, \mathrm{y}$ and $\mathrm{z}$ directions) and they support stress stiffening, large deflection and large strain capabilities. The structure was meshed with quadrilateral-shaped elements and clamped at one end. For modal analysis, the default Block Lanczos mode-extraction method was used. Model's mechanical parameters are summarized in Table 1 , assuming $C=1$ in (1). 
Table 1. Mechanical parameters used for FEM simulations.

\begin{tabular}{cccc}
\hline Properties & ANSYS parameters & Values for Si & Values for Porous Si \\
\hline Young's modulus (GPa) & EX, EY & 169 & $169 \times(1-p)^{2}$ \\
& EZ & 130 & $130 \times(1-p)^{2}$ \\
Poisson ratio & NUXY & 0.0625 & $0.0625 \times(1-p)$ \\
& NUYZ, NUXZ & 0.2785 & $0.2785 \times(1-p)$ \\
Shear modulus (GPa) & GXY & 50.85 & $50.85 \times(1-p)^{4}$ \\
& GYZ, GXZ & 79.51 & $79.51 \times(1-p)^{4}$ \\
Mass density $\left(\mathrm{kg} \cdot \mathrm{m}^{-3}\right)$ & DENS & 2320 & $2320 \times(1-p)$ \\
\hline
\end{tabular}

The comparison between analytical models and FEM results for different PS porosities is shown for three cantilever geometries that differ only by the beam thickness: $t_{b}=t_{p}=10 \mu \mathrm{m}, 25 \mu \mathrm{m}$ or $50 \mu \mathrm{m}$. Other common dimensions are: $L=2 \mathrm{~mm}$ and $w=500 \mu \mathrm{m}$ for the cantilever and $L_{S M}=w_{S M}=1 \mathrm{~mm}$ and $t_{S M}=450 \mu \mathrm{m}$ for the seismic mass. The evolution of the first eigenfrequency as a function of the porosity is plotted on Figure 2. Both models, (11) and (10), slightly overestimate the first eigenfrequency, but the Rayleigh's model gives values which are closest to those obtained by FEM. Eigenfrequency values calculated by (11) are between $1.24 \%$ (for the thinnest beam) up to $2 \%$ (the thickest beam) agreement with those obtained by FEM. These relative errors are, at least, about 1.5 times more important, when compared with the Timoshenko's model (the difference in both models is obvious only for the thickest cantilever in Figure 2).

For the thinnest cantilever which consists of $10 \mathrm{Si}$ on top of $10 \mathrm{PS}$, the simulated and calculated static deflections are plotted in Figure 3. The difference between the results obtained for cantilevers without mass (7) and cantilevers with mass (8) is undistinguishable (less than $2 \%$ ). Thus, the massless analytical model is in agreement within $2 \%$ with FEM simulations.

The simulated and calculated (9) longitudinal deformations $\epsilon_{L}$ on the surface of the cantilever are shown in Figure 4. For $L \leq x \leq L+L_{S M}$, the deformation is null: the hypothesis of a rigid seismic mass is confirmed by FEM.

\section{Experiments}

\subsection{Sample Preparation}

A series of devices were fabricated from a p-type (boron doped), 4 inches, double-side polished, (100)-oriented silicon wafer with a resistivity of $0.1-10 \Omega \cdot \mathrm{m}$. The process steps are summarized in Figure 5. On the frontside, a $300 \mathrm{~nm}$ aluminum layer was deposited by evaporation then patterned to define the geometry of the cantilevers ( $\mathrm{S} 1813$ photoresist and $\mathrm{Al}$ etchant). This was followed by a standard annealing for 30 minutes at $450^{\circ} \mathrm{C}$ in nitrogen ambient in order to form an Ohmic contact [15]. Cavities with a mesa were achieved on the backside of the wafer by Deep Reactive Ion Etching (DRIE) using a thick $(20 \mu \mathrm{m})$ AZ40XT photoresist mask. The nano-porous (pore width smaller than $2 \mathrm{~nm}$ ) 


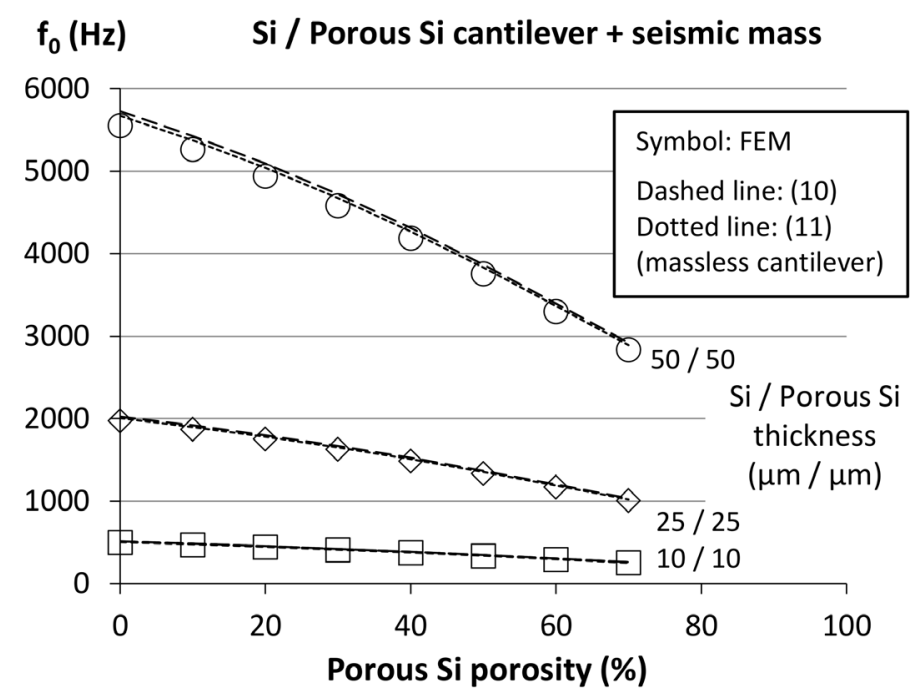

Figure 2. Evolution of the first eigenfrequency for three different $\mathrm{Si} /$ Porous Silicon cantilevers as a function of porosity. Common dimensions: $L=2 \mathrm{~mm}, w=500 \mu \mathrm{m}$. For the seismic mass: $L_{S M}=w_{S M}=1 \mathrm{~mm}$ and $t_{S M}=450 \mu \mathrm{m}$.

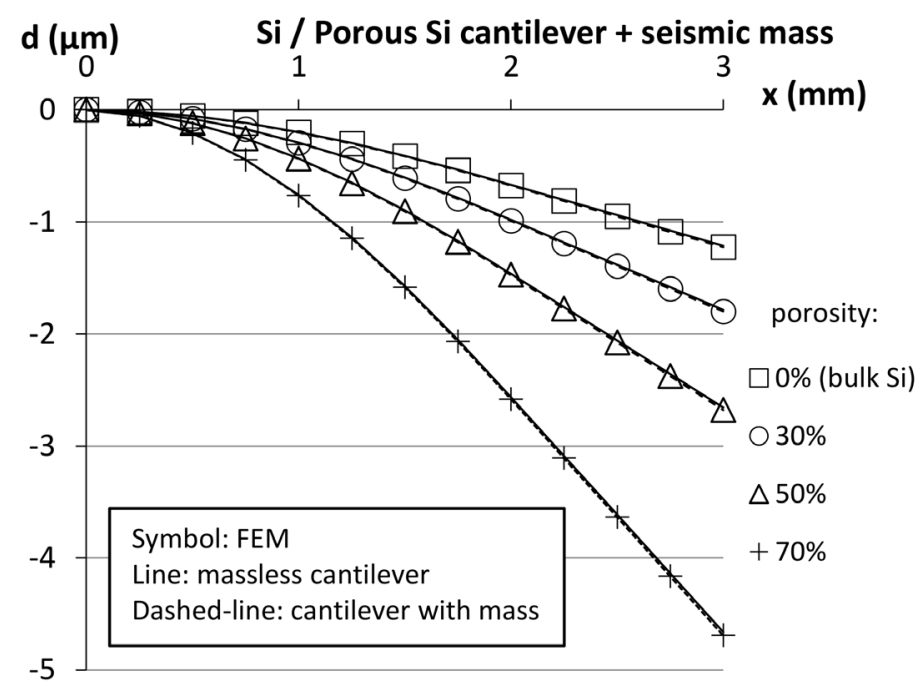

Figure 3. Static deflection of cantilevers for different Porous Silicon porosities $(10 \mu \mathrm{m} \mathrm{Si} / 10 \mu \mathrm{m}$ Porous $\mathrm{Si})$. Dimensions of cantilever and of the seismic mass are the same as in Figure 2.

silicon layer was formed on the backside of the obtained membranes by electrochemical etching (anodization) of silicon in a mixture of hydrofluoridric acid (HF) and ethanol (9 mL HF 48\% + $1 \mathrm{~mL}$ ethanol) for 20 minutes at a constant current density of $43 \mathrm{~mA} / \mathrm{cm}^{2}$. The aluminum pattern on the frontside of the wafer acted as a positive electrode for the anodization process of silicon. A platinum wire, in contact with the HF solution, was used as a negative electrode. Each membrane was processed individually. A picture of the silicon wafer during the anodisation process is shown in Figure 6. Several tentatives were made in order to define the right current density such that to obtain the PS formation 


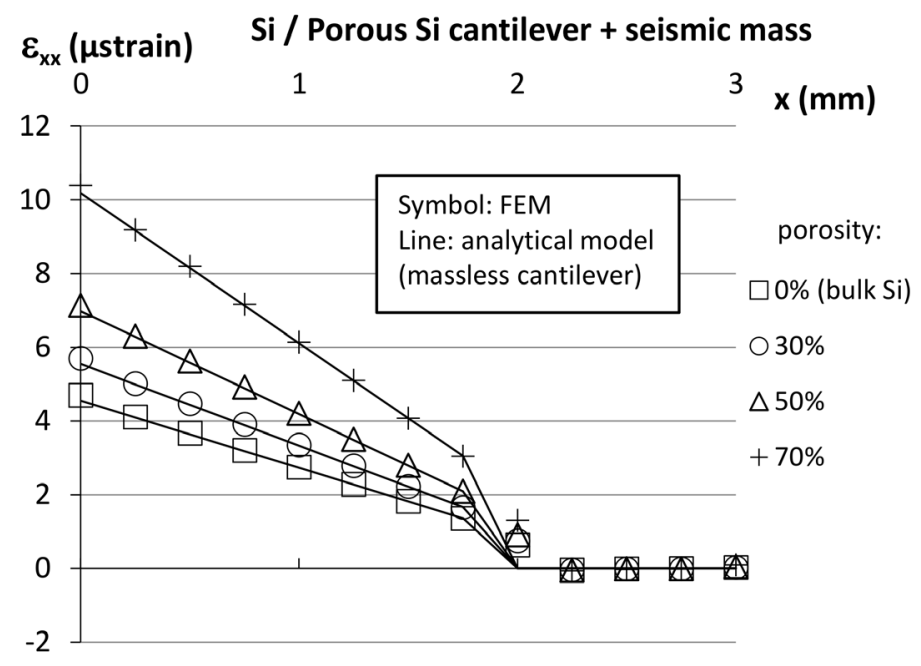

Figure 4. Longitudinal deformation on the cantilever surface. Dimensions: $2 \mathrm{~mm} \times 500 \mu \mathrm{m} \times 20 \mu \mathrm{m} \quad(10 \mu \mathrm{m} \mathrm{Si} / 10 \mu \mathrm{m}$ PS $)$. Seismic mass: $1 \mathrm{~mm} \times 1 \mathrm{~mm} \times 450 \mu \mathrm{m}$.

a)

$\begin{array}{ll}\square \mathrm{Si} & \square \text { Porous Silicon } \\ \mathrm{Al} & \square \text { AZ40XT }\end{array}$

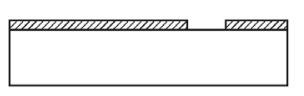

Double-side polished 4" (100) Si wafer + frontside $\mathrm{A} 1$ deposition $(300 \mathrm{~nm})+$ patterning + annealing $\left(450^{\circ} \mathrm{C} / 30 \mathrm{~min}\right)$

b)

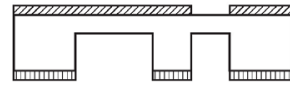

Backside Si DRIE (AZ40XT photoresist mask)

c)

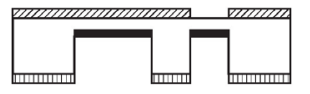

Porous Silicon formation

d)

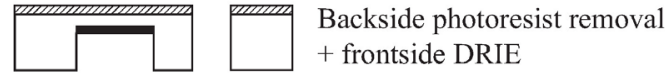

Figure 5. Process steps for the realization of Si and Si/Porous Si cantilevers.

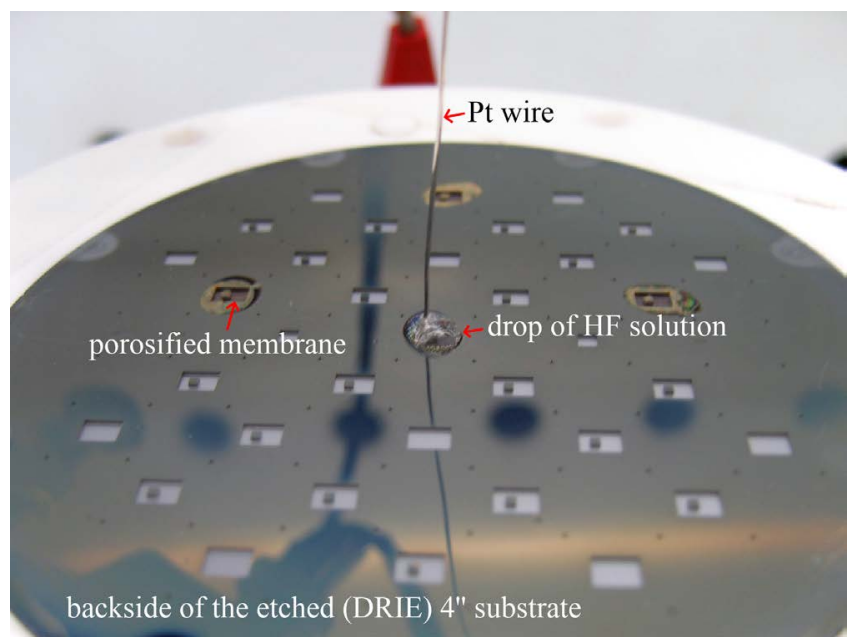

Figure 6. Backside of the micro-machined wafer with some porosified membranes-dark zones in the picture. 
regime and avoid the electropolishing regime. Finally, the $\mathrm{Al}$ patterns were used as a mask for a second Si DRIE step in order to release the cantilevers from the substrate. The bulk density of PS (density of the material including pores and interparticle voids [16]) was estimated to be about $50 \%$.

\subsection{Results}

After dicing, each sample was mounted on a vibrating pot and analyzed by Laser Doppler Vibrometry (Polytec MSA-500 micro-system analyzer). The measurement system and a close view of a sample are shown in Figure 7. The measured fundamental frequencies and quality factors for different Si cantilevers and one composite Si/PS cantilever are reported in Table 2. We obtained an average of the first eigenfrequency for the six silicon cantilevers: $(5447 \pm 120) \mathrm{Hz}$, and the quality factor $Q$ was measured to $(2870 \pm 400)$. For the Si/PS bilayer cantilever (sample \#1) the first measured eigenfrequency was $f_{0} \approx 5198 \mathrm{~Hz}$ with a quality factor $Q=1529$. The thickness of all cantilevers was measured at $(106 \pm 3) \mu \mathrm{m}$. By observing the side of the beam using a microscope for the Si/PS cantilever the

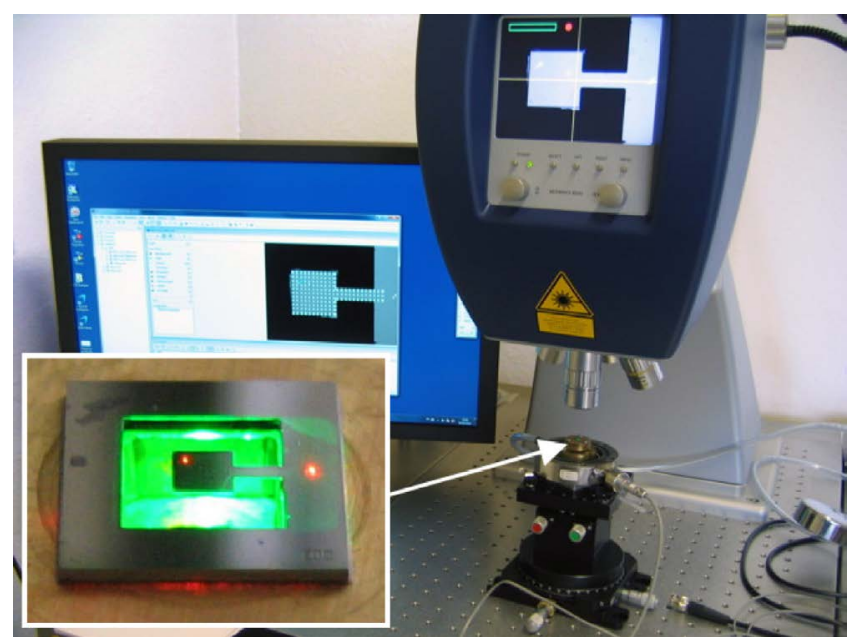

Figure 7. Characterization of a sample by laser Doppler vibrometry (Polytec MSA500).

Table 2. Experimental results on Si and Si/Porous Si cantilevers.

\begin{tabular}{cccccc}
\hline \multirow{2}{*}{ Sample \# } & \multirow{2}{*}{ Beam type } & \multicolumn{2}{c}{ with Al } & \multicolumn{2}{c}{ after Al removal } \\
\cline { 3 - 6 } & & $f_{0}(\mathrm{~Hz})$ & $Q$ & $f_{0}(\mathrm{~Hz})$ & $Q$ \\
\hline 1 & Si/Porous $\mathrm{Si}$ & 5198.4 & 1529 & & \\
2 & $\mathrm{Si}$ & 5471.6 & 2880 & 5464.1 & 3214 \\
3 & $\mathrm{Si}$ & 5567.2 & 2930 & & \\
4 & $\mathrm{Si}$ & 5501.6 & 3236 & & \\
5 & $\mathrm{Si}$ & 5334.6 & 2540 & & \\
6 & $\mathrm{Si}$ & 5366.4 & 3157 & & \\
7 & $\mathrm{Si}$ & 5442.3 & 2474 & 5434.4 & \\
Mean value & $\mathrm{Si}$ & $\approx 5447$ & $\approx 2870$ & & \\
\hline
\end{tabular}


PS thickness was measured as $(6.0 \pm 0.5) \mu \mathrm{m}$. The cantilever geometry was $L=1.3 \mathrm{~mm}$ and $w=400 \mu \mathrm{m}$, respectively while the seismic mass was estimated at $(1.7 \pm 0.1) \mathrm{mg}$. With that geometry, (11) leads to $f_{0}=5818 \mathrm{~Hz}$ for a Si cantilever and $f_{0}=5462 \mathrm{~Hz}$ for a Si/PS cantilever, which is about $5 \%$ agreement with measurements. The difference may be explained by geometrical uncertainties, particularly on the seismic mass.

We can conclude that, in agreement with the theoretical estimate, the first eigenfrequency of the Si/PS cantilever decreases by about $250 \mathrm{~Hz}$ compared to that of Si cantilevers (same cantilever thickness) while the quality factor was divided by almost a factor two. Measurements were also made on samples \#2 and \#7 after aluminum removal: the change in $f_{0}$ was limited to less than $0.2 \%$ whereas $Q$ increased by about $15 \%$. This shows that any non-uniformity of the aluminum thickness cannot explain the observed difference between the Si/PS sample and other Si samples, which is clearly the result of the stiffness decrease in the Si/PS sample with respect to bulk $\mathrm{Si}$.

\section{Conclusions}

The starting point of this work was the observation that the use of porous silicon in a bilayer cantilever may decrease the first eigenfrequency of the structure \{cantilever + seismic mass\}. The analytical estimate of this qualitative feature was firstly computed under classical Euler-Bernoulli one-dimensional bending model, neglecting the stiffness of the seismic mass and the mass contribution of the cantilever. Before comparison to measured data, the analytical results were confirmed by numerical results obtained by FEM under much general assumptions including three-dimensional elasticity and finite strains. Fabrication of a series of bulk Si and Si/PS cantilevers was performed and the measured eigenfrequencies show a good agreement with the predicted results. We notice also a decrease of the quality factor in that Si/PS bilayer, when compared to bulk $\mathrm{Si}$.

Among the open perspectives beyond this work we mention here first, the use of thick porous silicon layer which is expected to significantly decrease the eigenfrequencies of the system. The main drawback along path may be the mechanical resistance of the porous silicon. Second, the systematic study of the optimal geometries (mechanical and inertial properties of the seismic mass and cantilever) in order to improve quality factors at resonant frequencies may be of interest not only for energy harvesting application but also as a generic result.

\section{Acknowledgements}

The French National Research Agency (ANR) through PIEZO2POWER project has supported this work. Authors gratefully acknowledge facilities and technological staff's cooperation from NanoLyon platform. This work has been performed with the help of the "Plateforme Technologique Amont" of Grenoble, with the financial support of the "Nanosciences aux limites de la Nanoélectronique" Fundation and CNRS Renatech network. 


\section{Conflicts of Interest}

The authors declare no conflicts of interest regarding the publication of this paper.

\section{References}

[1] Roundy, S., Steingart, D., Frechette, L., Wright, P.K. and Rabaey, J.M. (2004) Power Sources for Wireless Sensor Networks. In: Karl, H., Wolisz, A. and Willig, A., Eds., Wireless Sensor Networks, EWSN 2004, Lecture Notes in Computer Science, Vol. 2920, Springer, Berlin, Heidelberg. https://doi.org/10.1007/978-3-540-24606-0_1

[2] Beeby, S.P., Tudor, M.J. and White, N.M. (2006) Energy Harvesting Vibration Sources for Microsystems Applications. Measurement Science and Technology, 17, R175-R195. https://doi.org/10.1088/0957-0233/17/12/R01

[3] Basu, S. and Kanungo, J. (2011) Nanocrystalline Porous Silicon, Crystalline Silicon-Properties and Uses. Prof. Sukumar Basu (Ed.), InTech.

http://www.intechopen.com/books/crystalline-silicon-properties-and-uses/nanocry stalline-porous-silicon

[4] Granitzer, P. and Rumpf, K. (2010) Porous Silicon-A Versatile Host Material Materials. Materials, 3, 943-998. https://doi.org/10.3390/ma3020943

[5] Stolyarova, S., Cherian, B., Raiteri, R., Zeravik, J., Skladal, P. and Nemirovsky, Y. (2008) Composite Porous Silicon-Crystalline Silicon Cantilevers for Enhanced Biosensing. Sensors and Actuators B, 131, 509-515. https://doi.org/10.1016/j.snb.2007.12.038

[6] Gaburro, Z., Daldosso, N. and Pavesi, L. (2005) Porous Silicon. In: Bassani, G.F., Liedl, G.L. and Wyder, P., Eds., Encyclopedia of Condensed Matter Physics, Vol. 4, Academic, 391-401.

[7] Garel, O., Breluzeau, C., Dufour-Gergam, E., Bosseboeuf, A., Belier, B., Mathet, V. and Verjus, F. (2007) Fabrication of Free-Standing Porous Silicon Microstructures. Journal of Micromechanics and Microengineering, 17, S164-S167. https://doi.org/10.1088/0960-1317/17/7/S13

[8] Bellet, D. (1997) Elastic Properties of Porous Silicon. In: Canham, L., Ed., Properties of Porous Silicon, INSPEC, London, 127-131.

[9] Gibson, L.J. and Ashby, M.F. (1997) Cellular Solids: Structure and Properties. Cambridge University Press, Cambridge. https://doi.org/10.1017/CBO9781139878326

[10] Altenbach, H. and Oechsner, A. (2011) Fracture Mechanics of Foams. In: Altenbach, H. and Öchsner, A., Eds., Cellular and Porous Materials in Structures and Processes. CISM Courses and Lectures, Vol. 521, Springer, New York, 1-46.

[11] Magoariec, H. and Danescu, A. (2009) Modeling Macroscopic Elasticity of Porous Silicon. Physica Status Solidi C, 6, 1680-1684.

https://doi.org/10.1002/pssc.200881053

[12] Timoshenko, S. and Young, D.H. (1961) Vibration Problems in Engineering. 3rd Edition, Van Nostrand, New York.

[13] Bhave, S. (2010) Mechanical Vibrations: Theory and Practice. Pearson Education, India.

[14] Roylance, L.M. and Angell, J.B. (1979) A Batch-Fabricated Silicon Accelerometer. IEEE Transactions on Electron Devices, D-26, 1911-1917. https://doi.org/10.1109/T-ED.1979.19795

[15] Sailor, M.J. (2012) Porous Silicon in Practice-Preparation, Characterization and Applications. Wiley-VCH, Weinheim, DE. 
[16] Rouquerol, J., Avnir, D., Fairbridge, C.W., Everett, D.H., Haynes, J.H., Pernicone, N., Ramsay, J.D.F., Sing, K.S.W. and Unger, K.K. (1994) Recommendations for the Characterization of Porous Solids. Pure and Applied Chemistry, 66, 1739-1758.

https://doi.org/10.1351/pac199466081739 